\title{
Origine des contraintes tectoniques déviatoriques dans la lithosphère terrestre
}

\author{
The sources of lithospheric tectonic stresses
}

\author{
L. FLEITOUT \\ Laboratoire de Géologie ENS ${ }^{*}$
}

Rev. Franç. Géotech. n56, pp. 43-50 (juillet 1991)

\section{Résumé}

Les contraintes associées aux déformations tectoniques de grande ampleur ont trois origines possibles : 1 . les forces appliquées en bout de plaque contrebalancées par la résistance visqueuse sous les plaques, 2. les hétérogénéités de densité à l'intérieur des plaques, à moins de $150 \mathrm{~km}$ de profondeur, 3. les hétérogénéités de densité situées profond dans le manteau terrestre. Dans le cas d'une convection à deux couches dans le manteau terrestre, cette dernière source de contrainte est moins importante que les deux premières.

\footnotetext{
Abstract

The stresses associated with large-scale tectonic deformation have three possible origins : 1 . the plate-boundary forces counter-balanced by the viscous drag beneath the plates, 2 . density heterogeneities situated within the plates (so at depth shallower than $150 \mathrm{~km}$ ), 3. deep mantelic mass heterogeneities. If the mantle convects in two layers, the deep mass anomalies appear to be less important than the other two sources of stress.
}

- 24, rue Lhomond, 75005 Paris. 


\section{INTRODUCTION}

Les grandes structures géologiques continentales (chaînes de montagnes, grabens et bassins), se forment par épaississement ou étirement des « plaques ». Ce sont aux contraintes qui induisent ces épaississements ou amincissements que nous allons nous intéresser dans cet article. Nous qualifierons ces contraintes de * tectoniques » par opposition aux contraintes flexurales ou thermiques, qui existent évidemment aussi dans les roches, mais qui ne peuvent être associées à de grandes déformations lithosphériques.

La lithosphère désigne la partie la plus externe de la terre située entre la sufrace et environ $100 \mathrm{~km}$ de profondeur. Elle est plus froide que le manteau sousjacent, et donc moins déformable. C'est en fait elle qui constitue les plaques. Elle est constituée d'une croûte de densité relativement faible $(2,7-2,9)$, d'environ $30 \mathrm{~km}$ d'épaisseur sous les continents et $5 \mathrm{~km}$ d'épaisseur sous les océans. La partie inférieure de la lithosphère constitue la partie la plus externe du manteau : elle a une composition pétrologique très proche de celle du manteau sous-jacent mais une densité légèrement plus élevée puisqu'elle est plus froide (la température varie de $0^{\circ} \mathrm{C}$ à environ $1200^{\circ} \mathrm{C}$ entre le haut et le bas de la lithosphère et le coefficient d'expansion thermique est voisin de $3.10^{-5} /{ }^{\circ} \mathrm{C}$. La densité du manteau, voisine de 3,3 , varie donc de quelques pourcents à cause des variations de température). Comme il sera montré dans la section 2, les anomalies de densité associées à des variations d'épaisseur de la croute ou de la lithosphère thermique sont les sources principales des variations de relief et modifient fortement le champ de contrainte.

Le relief, moyenné régionalement, est gouverné par l'isostasie : le poids d'une colonne verticale de matière prise entre la surface et la base de la lithosphère est partout le même:

$$
\int_{0}^{L} \Delta \rho d z=-\rho_{c} h
$$

où $\mathrm{h}$ désigne l'altitude au-dessus de la colonne considérée, L l'épaisseur maximale de la lithosphère et $\rho_{c}$ la densité de la croûte. Ainsi, au-dessus d'anomalies de densité négatives dans la lithosphère, il y a une élévation d'altitude de façon que (1) soit vérifiée. La figure 1 représente un schéma simple de lithosphères amincies et de lithosphères épaissies. Un épaississement crustal correspond à une anomalie négative de densité, mais l'épaississement de la partie inférieure de la lithosphère (constituée de manteau froid) correspond à une anomalie positive de densité (et vice-versa pour un amincissement lithosphérique). Compte tenu des différences de densité entre la croute, la lithosphère mantélique froide et le manteau sous-jacent, l'altitude est plus basse (graben) au-dessus d'une lithosphère amincie alors qu'elle est plus élevée (chaîne de montagne) au-dessus d'une lithosphère épaissie. Certaines montagnes ou certains plateaux sont dus à une lithosphère plus chaude sans épaississement de la croûte.

Seul le déviateur de contrainte est important pour la déformation lithosphérique. C'est donc à l'origine de ces contraintes déviatoriques que nous allons nous

\author{
EPAISSISSEMENT \\ DE LA LITHOSPHERE
}

\section{AMINCISSEMENT \\ DE LA LITHOSPHERE}

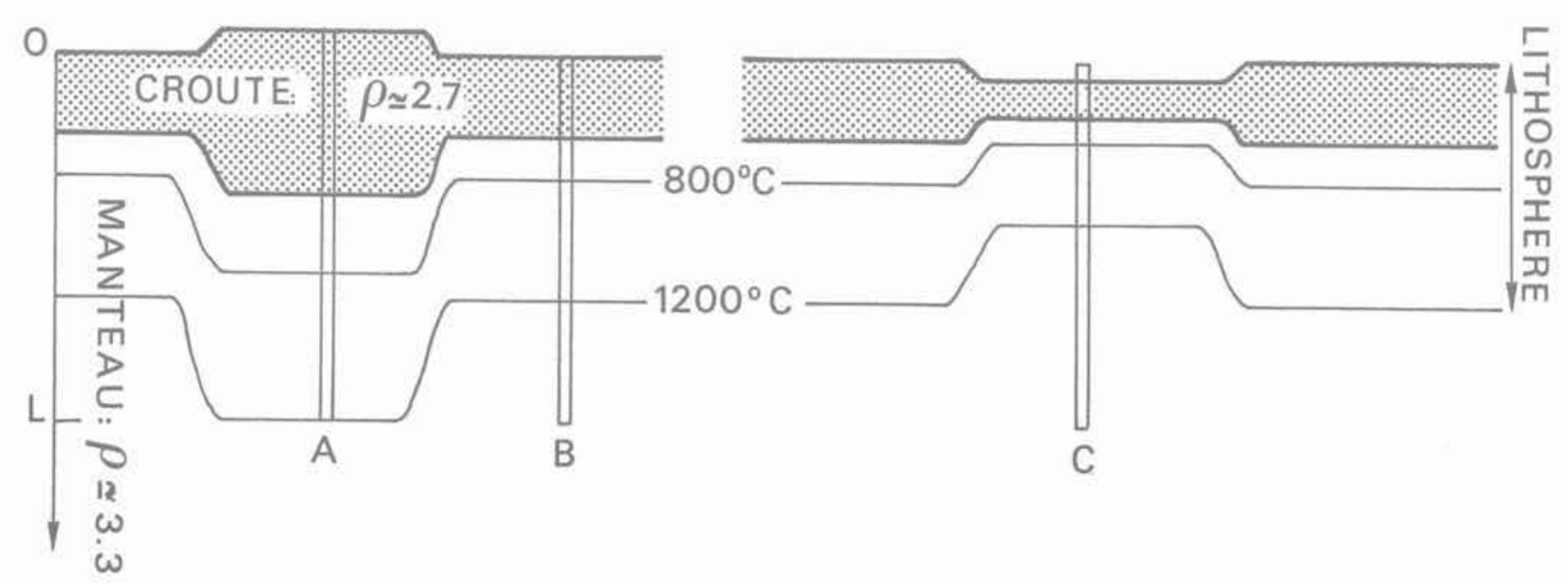

Fig. 1. - Schémas d'une lithosphère épaissie et d'une lithosphère amincie compensées.

L'isostasie implique que les poids de colonnes de la même base situées en A, B ou C sont égaux.

Fig. 1, - Schematic representation of thickened and thinned lithospheres. Isostasy implies equal weight for the columns A, B and C. 
intéresser. A grandes longueurs d'onde, c'est la quantité $\Sigma=\int_{0}^{L} \sigma_{x x}-\sigma_{z z} \mathrm{~d} z$ qui va gouverner la déformation. Nous noterons positivement les déviateurs d'extension. Nous dirons donc qu'il y a extension au sens géologique (étirement de la lithosphère) lorsque $\Sigma$ est positif. Notons que $\Sigma$ a pour unité des $\mathrm{Pa} . \mathrm{m}$ (ou kbar.km), comme les "forces " par unité de longueur décrites au paragraphe suivant.

\section{FORCES SUR LES PLAQUES}

La principale force qui meut les plaques est la conséquence de la subduction : c'est la traction des plaques plongeantes, denses qui entraînent vers elles à la fois la plaque océanique à laquelle elles sont attachées et la plaque chevauchante. La traction exercée par une plaque plongeante sur la plaque océanique est due à l'excès de poids de la plaque froide par rapport au manteau :

$$
\mathrm{P}=\iiint \Delta \rho \mathrm{g} \mathrm{d} z \mathrm{dx} d \mathrm{y}
$$

Pour une plaque de $700 \mathrm{~km}$ de long, ce poids est d'environ $410^{13} \mathrm{~Pa} . \mathrm{m}$ multiplié par $\delta y$ ( $\delta y$ désigne ici la largeur de la plaque dans la direction perpendiculaire au plan de la figure 2). Des forces de résistance visqueuses s'opposent à la pénétration de la plaque plongeante dans le manteau et supportent une partie importante du poids de la plaque plongeante. La force horizontale finalement disponible pour tirer

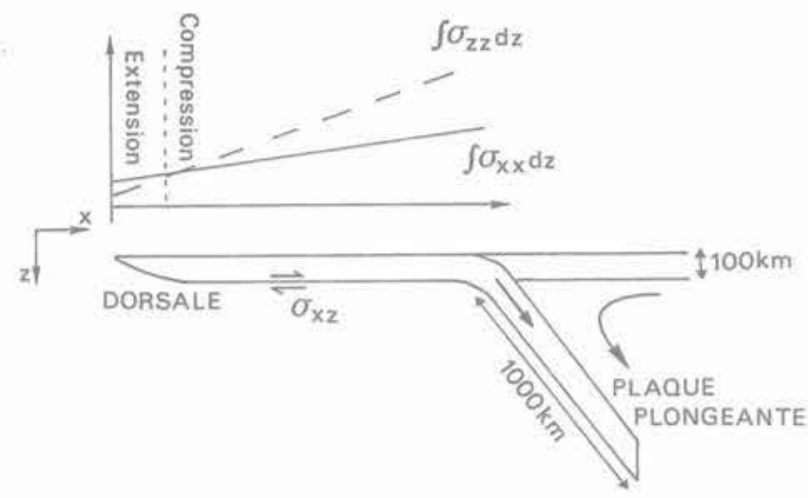

Fig. 2. - Schéma de l'évolution des contraintes dans une plaque océanique homogène tirée par une plaque plongeante. A cause du cisaillement visqueux sous la plaque, la contrainte horizontale croît de gauche à droite. La contrainte verticale dans la plaque non subductée croît également au fur et à mesure que la plaque se refroidit. La contrainte tectonique qui correspond à la différence entre contrainte horizontale et contrainte verticales (i.e. à la différence entre les courbes en trait plein et en pointillés sur le graphiquel correspond à de l'exten-

sion près de la dorsale et à de la compression loin de la dorsale.

Fig. 2. - Simplified evolution of the horizontal and vertical stresses in an oceanic lithosphere dragged by a slab. The shear stress associated with the viscous drag beneath the plate implies an increase of the horizontal stress away from the ridge. As the lithosphere cools (i.e. becomes denser) away from the ridge, the vertical stress also increases. The "tectonic stress " which corresponds to the difference between the horizontal and vertical stresses is observed to be extensional close to the ridge and compressive at older ages. la plaque est bien inférieure au poids de la plaque plongeante, vraisemblablement de l'ordre de $10^{12}$ Pa.m $\delta y$.

Le mouvement des plaques en surface induit un flux de matière dans le manteau sub-lithosphérique visqueux, accompagné d'une contrainte de cisaillement à la base des plaques. Cette contrainte de cisaillement correspond à une force résistant au mouvement de la plaque (fig. 2). Il existe plusieurs autres forces s'exerçant sur les plaques qui sont des forces d'interactions entre plaques, surtout résistantes : ce sont les forces correspondant au cisaillement au niveau des failles transformantes ou à la résistance à la collision entre plaques (FORSYTH et UYEDA, 1975). On inclut souvent dans les forces exercées sur les plaques la " poussée à la dorsale ». Cette dernière correspond en fait plutôt à une variation de contrainte verticale liée aux variations de densité thermiques dans la lithosphère (FLEITOUT et FROIDEVAUX, 1983) et sera discutée au paragraphe suivant.

La figure 2 présente un schéma simple d'une plaque plongeante et du cisaillement sous la plaque. La courbe en trait plein présente la variation de contrainte horizontale intégrée induite par le cisaillement sous la plaque: en effet, l'équation d'équilibre horizontal implique :

$$
\frac{\partial}{\partial \mathrm{x}} \int_{\mathrm{O}}^{\mathrm{L}} \sigma_{\mathrm{xx}} \mathrm{dz}=-\sigma_{\mathrm{xz}}
$$

Il sera montré au paragraphe suivant que la contrainte verticale varie aussi avec x (ligne tiretée sur la figure 2) et que donc la contrainte déviatorique moyennée $\int_{0}^{L} \sigma_{x x}-\sigma_{z z} d z$ ne peut être déterminée par par $\sigma_{\mathrm{xx}}$ seule.

Ces forces sur les plaques ont pendant longtemps été considérées comme la seule source de contraintes dans les plaques (RICHARDSON et al., 1979). Dans les paragraphes suivants nous allons montrer l'importance d'autres sources de contraintes. Cependant, ces forces sur les plaques, et en particulier la traction exercée par les plaques plongeantes, restent des éléments très importants du champ de contrainte. L'état de contrainte dans la plaque indienne est, en particulier, un bon exemple de l'importance de ces forces en bout de plaque. La subduction indonésienne tire vers le nord la plaque indienne. La collision continentale au niveau de l'Himalaya résiste à ce mouvement vers le nord. Il en résulte une compression importante nordsud dans le bassin du Bengale, avec par contre une tendance à l'extension perpendiculairement à la zone de subduction (CLOETINGH et WORTEL, 1985).

\section{ANOMALIES DE DENSITÉ DANS LA LITHOSPHÈRE}

Les forces sur les frontières de plaques contrebalancées par le frottement visqueux sous les plaques ne peuvent expliquer les brusques variations du champ de contrainte observées dans certaines régions telles que l'Europe. En effet, comme on verra à la fin de ce paragraphe, il existe de nombreux exemples de zone de compression et d'extension situées côte à côte à quelques centaines de kilomètres l'une de l'autre. Les variations de densité à l'intérieur de la lithosphère 
constituent des éléments importants du champ de contrainte qui permettent d'expliquer de telles variations de contraintes.

\subsection{La loi du «moment»}

Considérons une anomalie de masse surfacique $\Delta \mathrm{m}$, située dans la lithosphère à une profondeur $\mathrm{d}$. On veut comprendre comment elle influence le champ de contrainte. Des calculs rigoureux ont été effectués dans le cas de couches visqueuses par FLEITOUT et FROIDEVAUX (1982). Ici, nous présentons une démonstration simple basée sur des approximations valables « à grande longueur d'onde " i.e. nous allons nous placer dans le cas où l'extension horizontale $\mathrm{dx}$ de l'anomalie de masse est grande par rapport à L. l'épaisseur de la lithosphère. Dans ce cas, le taux de déformation horizontale $\dot{\epsilon}_{\mathrm{xx}}$ ne dépend pas de z. (La différence entre $\sigma_{\mathrm{xx}}$ et $\sigma_{\mathrm{zz}}$ se réajuste en fonction de la profondeur pour que $\dot{\epsilon}_{\mathrm{xx}}$ reste constant.) La vitesse et le signe de la déformation ne dépendent alors que de l'intégrale $\Sigma=\int_{0}^{L} \sigma_{\mathrm{xx}}-\sigma_{\mathrm{zz}} \mathrm{d} z$. L'équilibre horizontal de la plaque implique :

$\int_{0}^{\mathrm{L}} \sigma_{\mathrm{xx}} \mathrm{dz}=$ cte (l'influence de la contrainte de cisaillement en base de lithosphère dans l'équation (2) est négligeable sur de courtes distances horizontales). Si le style tectonique varie d'une région à une autre, ce ne peut donc être du aux variations de la contrainte horizontale.

Comment varie la contrainte verticale $\sigma_{z z}$ ? Nous allons examiner la différence de contrainte verticale entre une colonne loin de l'anomalie de densité $\delta \mathrm{m}$ (colonne B sur la figure 3 ) et une colonne contenant l'anomalie de masse (colonne A).

A la profondeur L, $\sigma_{z}$ est le même pour les deux colonnes (même pression dans le fluide qu'est le manteau sub-lithosphérique). L'équation d'équilibre vertical s'écrit :

$$
\frac{\partial \sigma_{z z}}{\partial z}+\frac{\partial \sigma_{x z}}{\partial x}=\Delta \rho \mathrm{g}
$$

où $\Delta \rho$ représente l'anomalie de densité locale.

A grande longueur d'onde, le terme $\partial \sigma_{x z} / \partial x$ peut être négligé. Donc $\partial \sigma_{z z} / \partial z$ est le même pour les deux colonnes sauf en $z=d$ :

$$
\partial\left(\sigma_{z z A}-\sigma_{z z B}\right) / \partial z=0 \text { pour } z \neq d
$$

$\sigma_{z z A}-\sigma_{z z B}$ présente une discontinuité égale à ômg en $z=\mathrm{d}$. La différence $\sigma_{z z A}-\sigma_{z B}$ correspond donc à la courbe tracée figure 3 . On en déduit :

$$
\int_{0}^{L} \sigma_{z z A}-\sigma_{z z B} d z=\delta m g d
$$

L'influence d'une anomalie de masse intralithosphérique sur le champ de contrainte est donc proportionnelle à son moment défini comme étant le produit de son amplitude par sa profondeur. (Ce terme a été choisi par analogie avec le *moment " d'un dipôle : l'anomalie de masse et la topographie qu'elle induit forment en effet un dipôle de masse de moment $\delta \mathrm{m}$ d.) Une anomalie de masse positive induit une compression $\left(\sigma_{z z}>\sigma_{x x}\right)$, et vice versa, une anomalie de masse négative induit de l'extension.

Notons qu'un relief égal à $-\delta \mathrm{m} / \rho$ se forme au-dessus de l'anomalie de masse $\delta \mathrm{m}$ (compensation isostatique, équation 1).

Par souci de simplicité, nous n'avons jusqu'à présent considéré qu'une anomalie de masse à une profondeur unique d. Il est évident que dans le cas d'une distribution d'anomalies de densité $\Delta \rho(\mathrm{z})$, la variation de contrainte verticale intégrée s'écrit:

$$
\int_{0}^{\mathrm{L}} \Delta \sigma_{z z} \mathrm{dz}=\mathrm{g} \int_{0}^{\mathrm{L}} \Delta \rho(\mathrm{z}) \mathrm{zdz}
$$
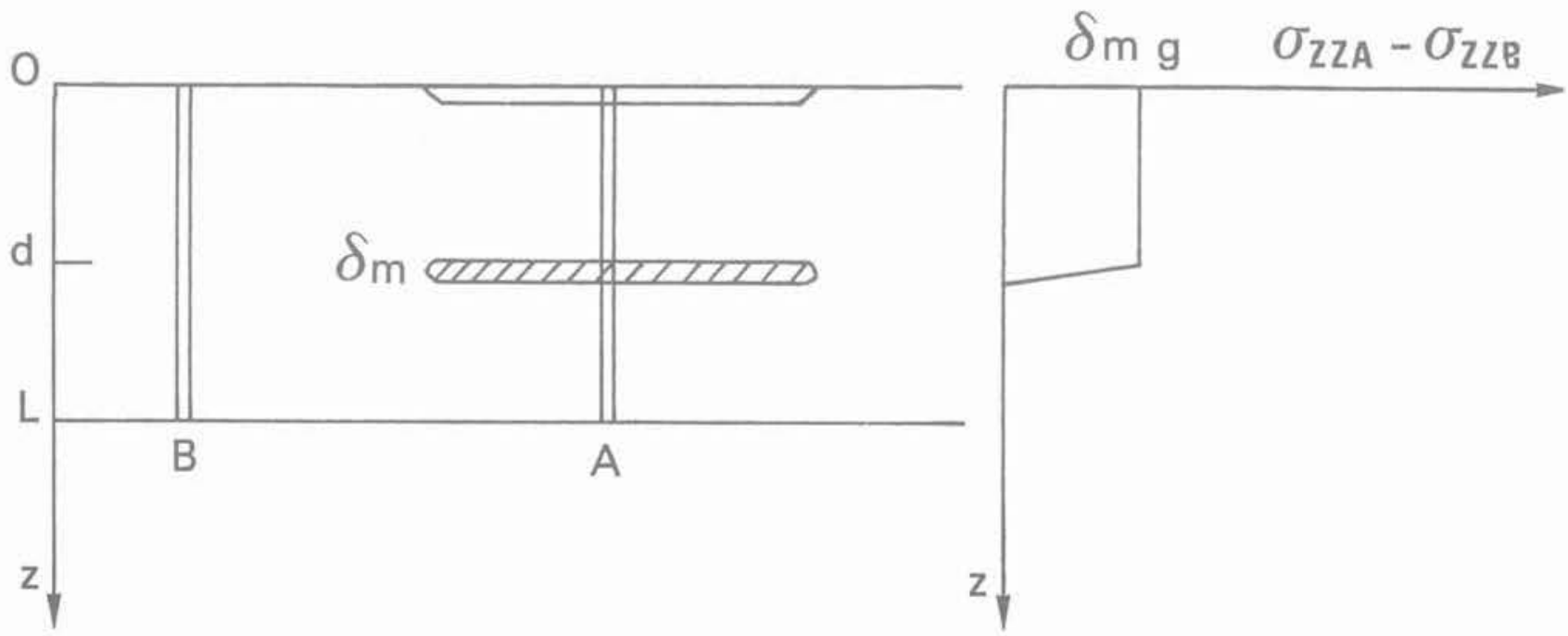

Fig. 3. - L'anomalie de masse óm située à la profondeur $d$ induit une variation de contrainte verticale égale à óm entre $d$ et la surface. L'effet de l'anomalie de masse ím est donc d'accroître $\int_{0}^{L} \sigma_{z z} d z d$ 'une quantité $\delta m g d$.

Fig. 3. - The mass anomaly óm located at a depth $d$ induces a vertical stress increase orm $g$ between $d$ and the Earth's surface. $\int_{0}^{L} \sigma_{z z} d z$ is in turn increased by $\delta m g d$. 
Selon l'équation (1), la variation de relief $\mathrm{h}$ est égale à :

$$
\mathrm{h}=-\int_{0}^{\mathrm{L}} \Delta \rho(\mathrm{z}) \mathrm{dz} / \rho_{\mathrm{c}}
$$

Les deux intégrales dans (6) et (7) n'ont pas forcément le même signe. Ceci est illustré sur la figure 4 où il $y$ a superposition d'une anomalie peu profonde, d'amplitude $-2 \Delta \mathrm{m}_{1}$ et d'une anomalie d'amplitude $\Delta \mathrm{m}_{1}$ trois fois plus profonde. Plaçons nous dans le cas où $\Delta \mathrm{m}_{1}$ est positive. L'anomalie de topographie est alors positive mais la contrainte est plus compressive dans la région des anomalies de masse. Le relief seul ne suffit donc pas à indiquer le signe des contraintes associées aux anomalies de densité lithosphériques. Nous verrons que cette constatation est importante pour comprendre l'état de contrainte au-dessus des chaînes de montagne.

Les raisonnements simples présentés dans ce paragraphe ne sont valables qu'à grandes longueurs d'onde. Des calculs basés sur la résolution de l'équation de Navier-Stokes dans le cas de couches visqueuses (FLEITOUT et FROIDEVAUX, 1982) montrent les limites du domaine d'application de cette formulation pour des longueurs d'onde voisines de l'épaisseur de la lithosphère.

\subsection{Quelques exemples géologiques}

Il a été expliqué au paragraphe 2 que la contrainte horizontale moyenne dans une lithosphère océanique croit avec la distance à la dorsale. Cependant, la plaque océanique se refroidit et devient de plus en plus épaisse au fur et à mesure que l'on s'éloigne de la dorsale. Ce refroidissement correspond à des anomalies de densité positives profondes, donc à une augmentation de $\int_{0}^{L} \sigma_{z z} \mathrm{dz}$ avec la distance à la dorsale. La structure thermique de la lithosphère en fonction de la distance de la dorsale est bien connue. Cela permet d'évaluer l'augmentation de $\int_{0}^{L} \sigma_{z z} \mathrm{dz}$ entre la

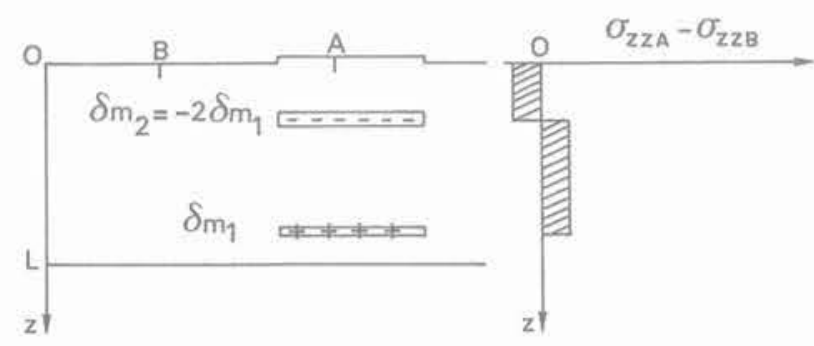

Fig. 4. - Malgré son relief élevé, la lithosphère en A est plus "en compression " qu'en B: le "moment " de l'anomalie de masse positive profonde $\delta m_{1}$ est supérieur au " moment " de l'anomalie négative $\delta m_{2} \cdot \int_{0}^{L} \sigma_{z z A}-\sigma_{z z B} d z$

(différence des aires hachurées à droite de la figure) est en effet positive.

Fig. 4. - Despite the fact that the relief is high around A, tectonic "compression" is stronger in A than in B: the "moment " of the positive deep mass anomaly $\delta m_{1}$ is larger than the "moment " of the negative shallower mass anomaly $\delta m_{2}$. Consequently, $\int_{0}^{L} \sigma_{z z A}-\sigma_{z z B} d z$ iwhich corresponds to the difference between the shaded areas on the left of the figure) is positive. dorsale et un vieil océan (appelée habituellement poussée à la dorsale) à environ $210^{12} \mathrm{~Pa}$.m. L'évolution de $\int_{0}^{\mathrm{L}} \sigma_{\mathrm{xx}}-\sigma_{z z} \mathrm{dz}$ en fonction de l'âge dépend de l'amplitude du cisaillement sous les plaques. Les données de contrainte dans les océans indiquent en fait que ce cisaillement est suffisamment faible pour que $\int^{\mathrm{L}} \sigma_{\mathrm{xx}}-\sigma_{z z} \mathrm{dz}$ décroisse avec la distance à la dorsale (FLEITOUT et FROIDEVAUX, 1983) : la contrainte déviatorique intégrée au niveau de la dorsale est une contrainte d'extension faible car la lithosphère $y$ est mince. En dehors des zones très proches des dorsales, les plaques océaniques sont presque toujours en compression comme le montrent les observations des mécanismes au foyer des séismes dans les plaques océaniques (SYKES et SBAR, 1974). Une telle évolution des contraintes déviatoriques implique que la contrainte verticale croît plus vite que la contrainte horizontale. Cette évolution des contraintes est schématisée sur la figure 2 .

Les zones de hauts plateaux comme les Andes ou le Tibet sont en extension généralement perpendiculaire à la direction de convergence, alors que l'épaississement lithosphérique semble continuer, autour de ces plateaux dans les zones de plus basse altitude. La figure 5 montre l'état de contrainte actuel dans les Andes d'après MERCIER et al. (1990). Cette variation du style tectonique d'est en ouest, s'explique facilement par une variation de la contrainte verticale moyenne $\sigma_{22}$. Les Andes ont à la fois une croûte plus épaisse et une lithosphère mantélique plus mince que les régions adjacentes qui sont en compression. L'épaississement crustal et l'amincissement lithosphérique correspondent à des anomalies de masses négatives, responsables à la fois de la haute altitude des Andes, et, par application de la loi du «moment ", de la tectonique en extension de la chaine de montagne. Ce régime tectonique a varié en fonction de la contrainte horizontale $\sigma_{\mathrm{EW}}$, qui selon MERCIER et al. (1990) change avec la forme de la plaque plongeante. Ainsi, à la fin du Miocène l'état de contrainte dans les Andes correspondait à $\sigma_{\mathrm{EW}}>\sigma_{\mathrm{NS}}>\sigma_{z z}$ (fig. 5).

La tectonique du Tibet et celle des Andes nous indiquent que lorsqu'un plateau atteint une altitude d'environ $4000 \mathrm{~m}$, la contrainte verticale intégrée sur l'épaisseur de la lithosphère devient inférieure à (plus compressive que) la contrainte horizontale. Comme dans ces deux cas, la contrainte verticale intégrée sur l'épaisseur de la lithosphère peut être estimée à environ $510^{12} \mathrm{~Pa} \cdot \mathrm{m}$, on a là une estimation des contraintes horizontales déviatoriques dans les plaques. Tout modèle qui prédit des contraintes horizontales dans les plaques très supérieures à cette quantité a de fortes chances d'être construit sur des hypothèses fausses.

Près de nous, le Massif Central avec les grabens de la Limagne et du Forez est une zone caractérisée par une extension $\mathrm{E}-\mathrm{W}$ et une compression $\mathrm{N}-\mathrm{S}$ alors que les Alpes sont une chaîne encore active, avec dans la partie française une compression E-W. L'altitude du Massif Central est plus faible que celle des Alpes. L'anomalie de densité négative totale sous les Alpes est donc plus grande en valeur absolue que sous le Massif Central. Cependant, la haute altitude du Massif Central est due non pas à un épaississement crus- 


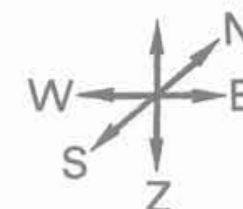

$\sigma_{\mathrm{ZZ}}>\sigma_{\mathrm{NS}}>\sigma_{\mathrm{EW}} \quad \sigma_{\mathrm{NS}}>\sigma_{\mathrm{EW}}>\sigma_{\mathrm{ZL}} \quad \sigma_{\mathrm{ZZ}}>\sigma_{\mathrm{NS}}>\sigma_{\mathrm{EW}}$

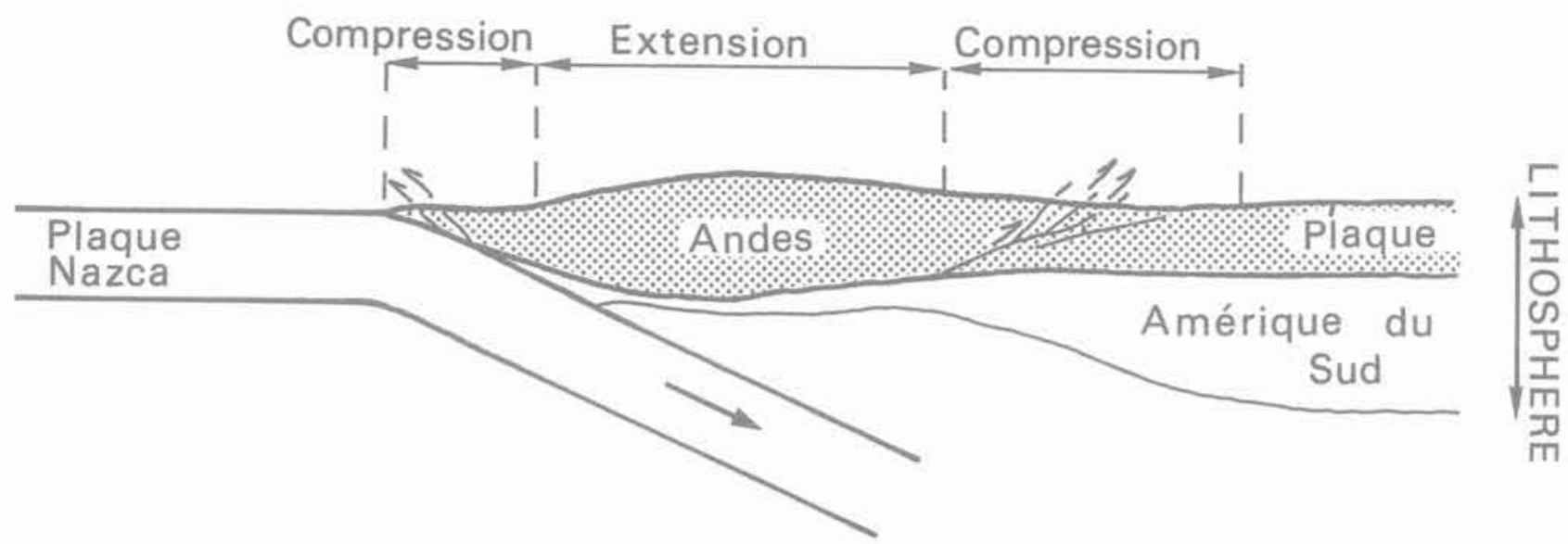

Fig. 5. - Schéma des contraintes dans les Andes (d'après MERCIER et al. 1990). Les Andes sont une zone de haute altitude compensée par des anomalies de masse négaitves en profondeur : croûte épaissie et lithosphère plus chaude. Ce sont ces anomalies de masse négatives profondes qui sont la cause de l'extension des plateaux Andins.

Fig. 5. - Schematic representation of the state of stress in the Andes (from MERCIER et al. 1990). The Andes correspond to a high altitude plateau. This plateau is compensated by low density material at depth : thickened crust or warmer lithosphere. This low density material induces extension in the Andes.

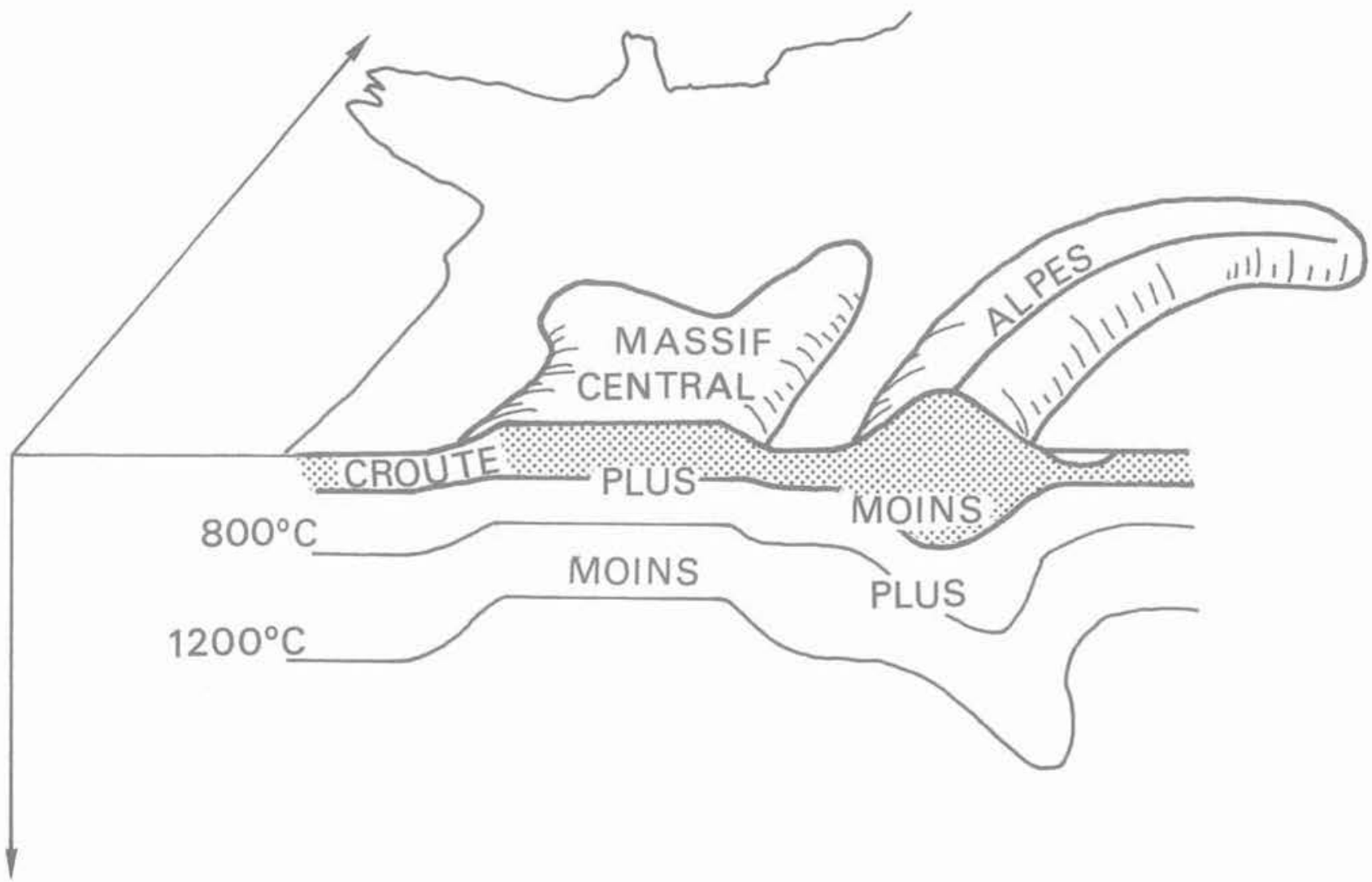

Fig. 6. - Coupe schématique à travers le Massif Central et les Alpes. Dans ce cas, épaississement crustal et épaississement de la lithosphère thermique correspondent à des anomalies de masse de signe opposé. Comme sur le schéma de la figure 4 , ce sont les anomalies les plus profondes qui ont l'influence tectonique prépondérante.

Fig. 6. - Schematic cross-section through the Alps and the Massif Central. Crustral thickening implies lighter material at depth while the thickening of the cold (dense) mantelic material forming the lower part of the lithosphere implies denser material at depth. As for figure 4, the deeper mass anomaly has a predominant tectonic influence. 
tral, mais à un réchauffement de la lithosphère, donc à une anomalie de densité négative profonde, à fort «moment ". L'altitude des Alpes est due à un épaississement crustral peu profond, vraisemblablement accompagné d'une anomalie de masse positive profonde correspondant à un épaississement lithosphérique (BABUSKA et al., 1984). Si l'on schématise la différence de densité entre les Alpes et le Massif Central, on a à faible profondeur une anomalie de masse négative sous les Alpes, positive sous le Massif Central. Cependant, à grande profondeur l'anomalie de masse est positive sous les Alpes et négative sous le Massif Central. On se trouve dans un cas semblable à celui de la figure 4 où l'anomalie de masse la plus profonde, bien que de plus faible amplitude, l'emporte par son * moment " et produit l'effet tectonique dominant. Notons qu'il y a de nombreux exemples de chaînes de montagne en compression à côté de bassins en extension (Toscane et Appenins, Bassin Pannonnier et Carpathes...).

\section{ANOMALIES PROFONDES DE DENSITÉ}

La * loi du moment * indique que plus une anomalie de densité se trouve profondément enfouie dans la lithosphère, plus elle influence le champ de contrainte lithosphérique. Qu'en est-il des anomalies de masse sub-lithosphériques? Depuis quelques années, la tomographie sismique permet de détecter des variations latérales de vitesse sismique dans le manteau terrestre à des profondeurs entre 100 et $3000 \mathrm{~km}$. Ces anomalies sismiques correspondent à des anomalies profondes de densité.

Ces anomalies de densité profonde ont-elles une influence très forte sur le champ de contrainte? La réponse à cette question dépend de la structure convective du manteau en une couche ou en deux couches.

4.1. Si le manteau convecte en une seule couche, en calculant, par résolution de l'équation de NavierStokes, l'écoulement du matériau visqueux entraîné par ces anomalies de masse, on montre que des contraintes déviatoriques très importantes (supérieures à $10^{13} \mathrm{~Pa} . \mathrm{m}$ ) peuvent être induites dans la lithosphère par les anomalies de densité du manteau inférieur (RICARD et al., 1984). Des variations de relief importantes, de l'ordre de $\pm 1 \mathrm{~km}$ devraient aussi être associées à ces anomalies de densité profondes.

$\mathrm{Si}$ le manteau convecte en deux couches, les anomalies de densité entraînent une déflexion de l'interface à $670 \mathrm{~km}$ mais la lithosphère se trouve mécaniquement découplée du manteau inférieur. Ni contraintes tectoniques importantes, ni reliefs ne doivent être dans ce cas associés aux anomalies de densité du manteau inférieur.

Aucune anomalie de relief n'est observée au-dessus des anomalies de densité du manteau inférieur (COLIN et FLEITOUT, 1990). Pour cette raison, lauteur du présent article préfère le modèle de convection à deux couches où la lithosphère est mécaniquement découplée du manteau inférieur par la dis- continuité à 670 km. Dans ce cas, les anomalies profondes de densité ne devraient pas constituer une source importante de contraintes.

\section{CONCLUSION}

Les forces exercées sur les frontières de plaques (en particulier la traction des plaques subductées), la résistance visqueuse au mouvement de la plaque, et les anomalies de densité à l'intérieur des plaques sont les principales sources du champ de contrainte.

Cet article ne traite que des sources du champ de contrainte. Ces contraintes agissent sur un milieu toujours hétérogène. La déformation est souvent concentrée sur des failles. Ces hétérogénéités mécaniques de la lithosphère influent évidemment sur le champ de contrainte local. Elles apparaissent comme un élément supplémentaire qu'il est important d'introduire dans les modèles de champ de contraintes.

\section{BIBLIOGRAPHIE}

BABUSKA V., PLOMERAVA J., SILENY J. (1984), Large scale oriented structures in the subcrustal lithosphere of Central Europe. Annales Geophysicae, 2-16, 117-123.

CLOETINGH S.A.P.L., WORTEL M.J.R. (1985), Regional stress field of the Indian plate GRL, 12-2 77-80.

COLIN P., FLEITOUT L. (1990), Topography of the ocean floor: thermal evolution of the lithosphere and interaction of deep mantle heterogeneities with the lithosphere GRL. 17-11 1961-1964.

FLEITOUT L., FROIDEVAUX C. (1982), Tectonics and topography for a lithosphère containing density heterogeneities. Tectonics, 1, 21-56.

FLEITOUT L., FROIDEVAUX C. (1983), Tectonic stresses in the lithosphere Tectonic, Vol. 12, $n^{\circ} 3,315-324$

FORSYTH D., UYEDA S. (1975), On the relative importance of the driving forces of plate motion Geophys, J.R. Astron. Soc., 43, 163-200.

MERCIER J.L., SEBRIER M., LAVENUE A., CABRERA J., BELLIER O., DUMONT J.F., MACHARE J., Changes in tectonic regime above a subduction zone of andean type: the Andes of Peru and Bolivia during the pliocene-pleistocene (JGR in press).

RICARD Y., FLEITOUT L., FROIDEVAUX C. (1984), Geoid heights and lithospheric stresses for a dynamid earth. Annales Geophysicae, Vol. 2, 267-286.

RICHARDSON R.M., SOLOMON S.C., SLEEP N.H. (1979), Tectonic stresses in the plates. Rev. Geophys. and Space Phys., 17, 981-1019.

SYKES L.R., SBAR M.L. (1974), Focal mechanism solutions of intraplate earthquakes and stresses in the lithosphere in "Geodynamics of Iceland and the North Atlantic Area » edited by L. Kristjansson, 207-224, Reidel Dordrecht. 


\section{CONVECTION SUR UNE SEULE COUCHE}

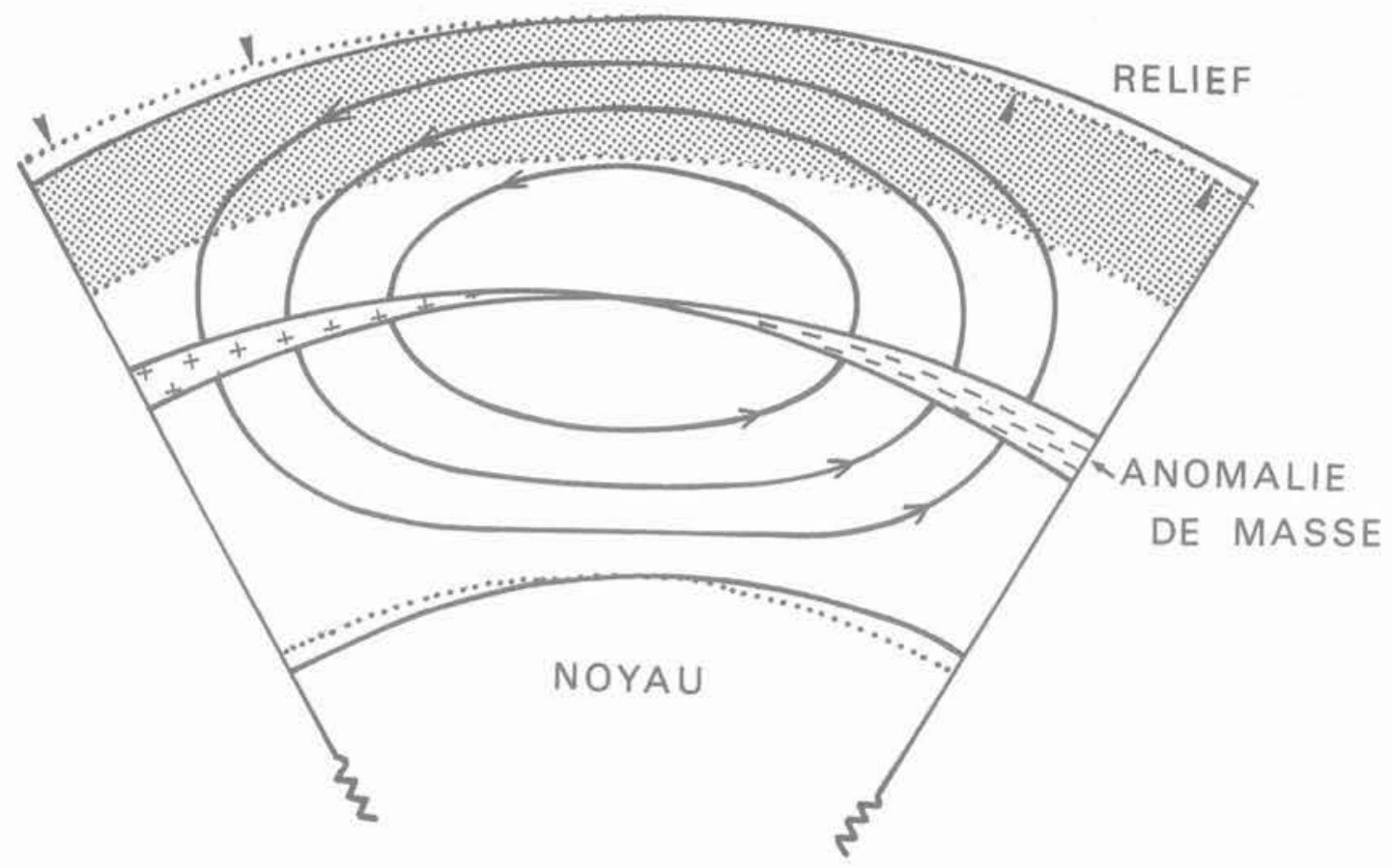

CONVECTION SUR DEUX COUCHES

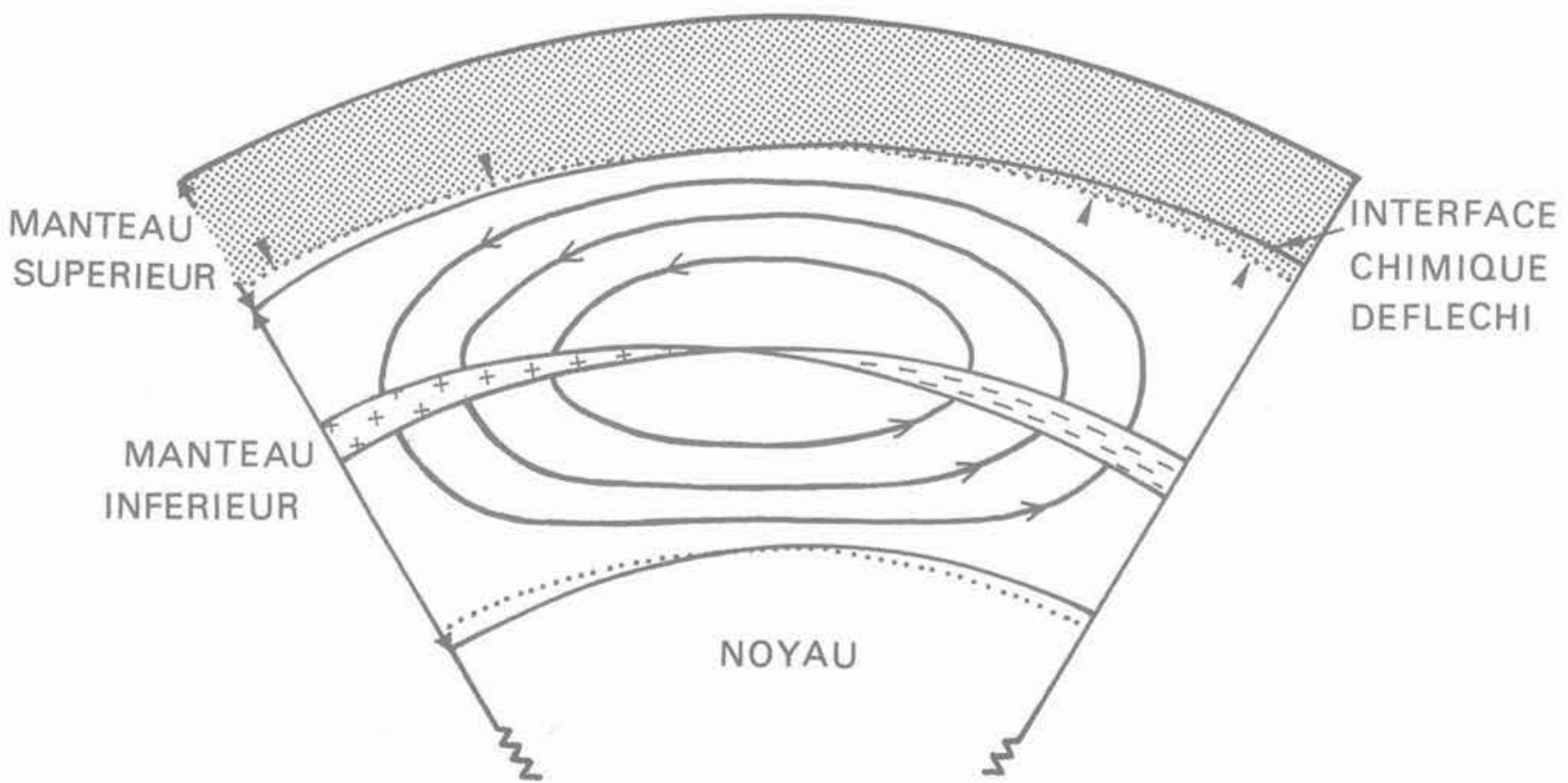

Fig. 7. - Schéma montrant l'influence possible d'anomalies de densité profondes sur les contraintes lithosphériques. Si le manteau convecte en une seule couche, les anomalies de densité du manteau inférieur peuvent induire à la fois d'importantes contrantes lithosphériques de l'ordre de $10^{8} \mathrm{~Pa}$ pour une lithosphère de $100 \mathrm{~km}$ d'épaisseur et des anomalies topographiques pouvant atteindre $\pm 1 \mathrm{~km}$. Si le manteau convecte en deux couches, la lithosphère se trouve mécaniquement isolée du manteau inférieur. Ni anomalies de relief, ni contraintes tectoniques ne sont alors associées aux anomalies de densité du manteau inférieur. Fig. 7. In the lithospheric stresses. For one layer mantle convection, the Fig. 7. - Influence of deep mantelic density heterogeneities on the lithospheric stresses. For one layer mantie $8.9 \mathrm{~Pa} \times 100 \mathrm{~km}$ and mantle mass heterogeneities induce huge lithospherice stresses and topography anomalies lof the order of $10 . \mathrm{Pa} \times 100 \mathrm{~km}$ and $\pm 1 \mathrm{~km}$ respectively). For two layer mantle convection, the lithosphere is mechanically decoupled from 